\title{
GEODESIC EXCURSIONS INTO AN EMBEDDED DISC ON A HYPERBOLIC RIEMANN SURFACE
}

\author{
ANDREW HAAS
}

\begin{abstract}
We calculate the asymptotic average rate at which a generic geodesic on a finite area hyperbolic 2-orbifold returns to an embedded disc on the surface, as well as the average amount of time it spends in the disc during each visit. This includes the case where the center of the disc is a cone point.
\end{abstract}

\section{INTRODUCTION}

A finite area hyperbolic 2-orbifold $S$ is the quotient of the Poincaré upper-half plane $\mathbb{H}$ by a discrete group $\Gamma \subset \mathrm{PSL}_{2}(\mathbb{R})$. Let $\pi: \mathbb{H} \rightarrow S=\mathbb{H} / \Gamma$ denote the covering projection and let $q \in S$ be a non-cone point of $S$. Without loss of generality, suppose that $i \in \mathbb{H}$ is a preimage of $q$. As usual, $B_{r}(x)$ denotes the open disc of radius $r$ centered at $x$. There is a number $R>0$ for which the map $\pi: B_{R}(i) \rightarrow B_{R}(q)$ is an embedding.

Each vector $v$ in the unit tangent bundle $T_{1} S$ uniquely determines a geodesic ray $\gamma=\gamma(v)$ which is the projection of the orbit of the geodesic flow, $\left\{G_{t}(v) \mid t \in[0, \infty)\right\}$, to $S$. If the ray keeps returning to the disc $B_{r}(q)$, then there is a sequence of pairs of parameter values $\left\{\left(t_{i}, s_{i}\right)\right\}$ with $t_{i}<s_{i}<t_{i+1}$, so that

$$
\bigcup_{i=1}^{\infty} \gamma\left[\left(t_{i}, s_{i}\right)\right]=\gamma \cap B_{r}(q) .
$$

In other words, $\gamma$ meets the disc $B_{r}(q)$ in precisely the $\operatorname{arcs} \gamma\left[\left(t_{i}, s_{i}\right)\right]$ of $\gamma$. We shall refer to these arcs as the excursions of $\gamma$ into $B_{r}(q)$. For $v \in T_{1} S$ define the counting function $N_{v}(r)(t)=\max \left\{i \mid t_{i} \leq t\right\}$. Then we have

Theorem 1. For almost all $v \in T_{1} S$ and all $0<r<R$,

$$
\lim _{t \rightarrow \infty} \frac{1}{t} N_{v}(r)(t)=\frac{2 \sinh r}{\operatorname{area}(S)}
$$

Two consequences of the theorem are given as follows.

Corollary 1. For almost all $v \in T_{1} S$ and all $0<r<R$, we have the following.

(i) The asymptotic average length of an excursion is

$$
\lim _{n \rightarrow \infty} \frac{1}{n} \sum_{i=1}^{n}\left(s_{i}-t_{i}\right)=\frac{\pi(\cosh r-1)}{\sinh r} .
$$

Received by the editors April 29, 2008.

2000 Mathematics Subject Classification. Primary 30F35, 32Q45, 37E35, 53D25.

Key words and phrases. Hyperbolic surface, Fuchsian group, geodesic flow.

(C)2009 American Mathematical Society 
(ii) The average length of an arc joining the beginnings of consecutive excursions is

$$
\lim _{n \rightarrow \infty} \frac{1}{n} \sum_{i=1}^{n-1}\left(t_{i+1}-t_{i}\right)=\frac{\operatorname{area}(S)}{2 \sinh r} .
$$

Note that when $r$ is large, (1.2) is close to $\pi$. In Section 3 we expand these results to cover the case where $q$ is a cone point. We will also consider their expressions in terms of the area of a disc rather than its radius.

\section{Details}

The unit tangent bundle of $\mathbb{H}$ is $T_{1} \mathbb{H}=\mathbb{H} \times S^{1}$. In these coordinates the geodesic flow $\tilde{G}$ has the natural invariant measure $\tilde{m}=d A d \theta$. The non-vertical vectors in $T_{1} \mathbb{H}$ are a set of full measure and can be parameterized by the set of triples $(x, y, t) \in \mathbb{R}^{3}$ with $x \neq y$. Let $\alpha$ be the geodesic in $\mathbb{H}$ oriented from the endpoint $\alpha_{-}=y$ to $\alpha_{+}=x$ and parameterized so that $\alpha(0)$ is the Euclidean midpoint of the semicircle $\alpha(\mathbb{R})$. Then $(x, y, t)$ corresponds to the vector $v=\dot{\alpha}(t) \in T_{1} \mathbb{H}$. In these coordinates the geodesic flow has the form $\tilde{G}_{s}(x, y, t)=(x, y, t+s)$. Furthermore, the geodesic flow on $T_{1} S$ has the invariant probability measure $\mu$, whose lift to $T_{1} \mathbb{H}$ is equal to

$$
\tilde{\mu}=\frac{1}{2 \pi \operatorname{area}(S)} \tilde{m}=\frac{1}{\pi \operatorname{area}(S)} \varphi(x, y) d x d y d t .
$$

where $\varphi(x, y)=(x-y)^{-2}$ ].

Let $\mathcal{E} \subset T_{1} S$ be the set of vectors $v$ for which there are infinitely many excursions of $\gamma=\gamma(v)$ into $B_{r}(q)$ for each $0<r<R$. As a consequence of the ergodic theorem and the Poincaré recurrence theorem, $\left[3, \mathcal{E}\right.$ has full measure in $T_{1} S$. Observe that if $v \in \mathcal{E}$, then $G_{t}(v) \in \mathcal{E}$ for all $t \in \mathbb{R}$.

Let $\mathcal{L}_{r}^{*}$ be the subset of the unit tangent bundle over the circle $\partial B_{r}(q)$ (bounding the disc $\left.B_{r}(q)\right)$ consisting of vectors that point into the $\operatorname{disc} B_{r}(q)$. Then $\mathcal{L}_{r}=\mathcal{L}_{r}^{*} \cap \mathcal{E}$ is a cross-section for the geodesic flow on $T_{1} S$, 2]. In other words, for almost all $v \in T_{1} S$ there exists an increasing sequence of values $\tau_{i}$ so that $G_{\tau_{i}}(v) \in \mathcal{L}_{r}$. Given $\epsilon<R-r$, define the $\epsilon$-thickened section $\mathcal{L}_{r}(\epsilon)=\left\{G_{t}(v) \mid t \in[0, \epsilon], v \in \mathcal{L}_{r}\right\}$. Analysis of the thickened section is the main tool in the proof of Theorem 1 This is similar to the approaches taken in [5], [6] and [8].

\section{Proposition 1.}

$$
\mu\left(\mathcal{L}_{r}(\epsilon)\right)=\frac{2 \epsilon \sinh r}{\operatorname{area}(S)} .
$$

The cross-section $\mathcal{L}_{r}$ lifts to a subset $\tilde{\mathcal{L}}_{r}$ of $T_{1} \mathbb{H}$ over the circle $\partial B_{r}(i)$ in $\mathbb{H}$. Then $\tilde{\mathcal{L}}_{r}(\epsilon)$ is a connected preimage of $\mathcal{L}_{r}(\epsilon)$ in $T_{1} \mathbb{H}$. Since $\pi$ is an embedding of $B_{r}(i)$ into $S$ and $\epsilon<R-r$, the projection $\pi_{*}: T_{1} \mathbb{H} \rightarrow T_{1} S$ restricts to an embedding of $\tilde{\mathcal{L}}_{r}(\epsilon)$ onto $\mathcal{L}_{r}(\epsilon)$. Thus $\mu\left(\mathcal{L}_{r}(\epsilon)\right)=\tilde{\mu}\left(\tilde{\mathcal{L}}_{r}(\epsilon)\right)$ and we can perform computations in $\mathbb{R}^{3}$ using the coordinates given earlier.

Define the Möbius transformations:

$$
W_{r}(x)=\frac{x \sinh r-1}{x+\sinh r} \quad \text { and } \quad U_{r}(x)=\frac{x \sinh r+1}{-x+\sinh r} .
$$

For real numbers $a \neq b$, let $\overline{a b}$ denote the geodesic $\alpha$ in $\mathbb{H}$ with endpoints $\alpha_{+}=a$ and $\alpha_{-}=b$. 
We will need the following extension of Theorem 5 from 4 .

Theorem 2. Given $x \geq 0$, the geodesics $\overline{x w}$ and $\overline{x u}$ with $w=W_{r}(x) \in[-1 / x, x)$ and $u=U_{r}(x)$, are both tangent to the disc $B_{r}(i)$. Furthermore, given $x \leq 0$, the geodesics $\overline{x w}$ and $\overline{x u}$ with $w=-W_{r}(-x) \in(x,-1 / x]$ and $u=-U_{r}(-x)$, are both tangent to the disc $B_{r}(i)$.

Although the proof in [4] only addressed the tangent geodesic with endpoints $x$ and $W_{r}(x)$, the other case can be uncovered there and follows by choosing $\xi=$ $z\left(c+i \sqrt{1-c^{2}}\right)$ in Lemma 3 and $\eta=c+i \sqrt{1-c^{2}}$ in the proof of Theorem 5.

Proof of Proposition 1. Fix $0<r<R$. For a given $x \in \hat{\mathbb{R}}$, let $I_{x}$ denote the set of values $y \in \hat{\mathbb{R}}$ for which the geodesic $\overline{x y}$ intersects the closed disc $\bar{B}_{r}(i)$. It follows from Theorem 2 that for $x \geq 0, I_{x}$ is the interval between the points $W_{r}(x)$ and $U_{r}(x)$; the unbounded interval if $x \leq \sinh \frac{r}{2}$ and the bounded interval if $x>\sinh \frac{r}{2}$. A similar statement is true for negative $x$.

For $x \in \mathbb{R}$ and $y \in I_{x}$, let $t_{x y}$ denote the parameter value for which the unit tangent vector $\left(x, y, t_{x y}\right) \in \tilde{\mathcal{L}}_{r}$. Then

$$
\tilde{\mathcal{L}}_{r}(\epsilon)=\left\{(x, y, t) \mid x \in \mathbb{R}, y \in I_{x} \text { and } t_{x y} \leq t \leq t_{x y}+\epsilon\right\} .
$$

Consequently,

$$
\begin{aligned}
\tilde{\mu}\left(\tilde{\mathcal{L}}_{r}(\epsilon)\right) & =\frac{1}{\pi \operatorname{area}(S)} \int_{\mathbb{R}} \int_{I_{x}} \int_{t_{x y}}^{t_{x y}+\epsilon} \varphi(x, y) d t d y d x \\
& =\frac{\epsilon}{\pi \operatorname{area}(S)} \int_{-\infty}^{\infty} \int_{I_{x}} \varphi(x, y) d y d x
\end{aligned}
$$

This unpacks as the following sum of integrals:

$$
\begin{aligned}
& \frac{\epsilon}{\pi \operatorname{area}(S)}\left[\int_{0}^{\sinh \frac{r}{2}} \int_{-\infty}^{W_{r}(x)} \frac{1}{(x-y)^{2}} d y d x\right. \\
& \quad+\int_{0}^{\sinh \frac{r}{2}} \int_{U_{r}(x)}^{\infty} \frac{1}{(x-y)^{2}} d y d x \\
&+\int_{\sinh \frac{r}{2}}^{\infty} \int_{U_{r}(x)}^{W_{r}(x)} \frac{1}{(x-y)^{2}} d y d x \\
&+\int_{-\sinh \frac{r}{2}}^{0} \int_{-W_{r}(-x)}^{\infty} \frac{1}{(x-y)^{2}} d y d x \\
&+\int_{-\sinh \frac{r}{2}}^{0} \int_{-\infty}^{-U_{r}(-x)} \frac{1}{(x-y)^{2}} d y d x \\
&\left.+\int_{-\infty}^{-\sinh \frac{r}{2}} \int_{-W_{r}(-x)}^{-U_{r}(-x)} \frac{1}{(x-y)^{2}} d y d x\right] .
\end{aligned}
$$

The integrals are easily computed.

Proof of Theorem 1. Let $\chi_{Y}(t)$ denote the characteristic function of the set $Y$. Then for $\epsilon<R-r$ we have the inequalities

$$
\int_{0}^{t} \chi_{\mathcal{L}_{r}(\epsilon)}\left(G_{\tau}(v)\right) d \tau-2 \epsilon \leq \epsilon N_{v}(r)(t) \leq \int_{0}^{t} \chi_{\mathcal{L}_{r}(\epsilon)}\left(G_{\tau}(v)\right) d \tau+2 \epsilon
$$


Divide the inequalities by $\epsilon t$. Since the geodesic flow is ergodic, as $t \rightarrow \infty$ the left and right hand limits approach $(1 / \epsilon) \mu\left(\mathcal{L}_{r}(\epsilon)\right)$. The theorem then follows from Proposition 1.

Proof of Corollary 1. First we address part (ii). Let $v \in \mathcal{E}$ and let $\left\{\left(t_{i}, s_{i}\right)\right\}$ be the sequence of excursions into the disc $B_{r}(q)$ associated to the geodesic $\gamma=\gamma(v)$. In particular, we have $N_{v}(r)\left(t_{n}\right)=n$ (or $n-1$ if $\gamma(0) \in B_{r}(q)$ ). Then by Theorem 1, for almost all $v \in T_{1} S$,

$$
\lim _{n \rightarrow \infty} \frac{1}{n} \sum_{i=1}^{n-1}\left(t_{i+1}-t_{i}\right)=\lim _{n \rightarrow \infty} \frac{t_{n}}{n}=\lim _{n \rightarrow \infty} \frac{t_{n}}{N_{v}(r)\left(t_{n}\right)}=\frac{\operatorname{area}(S)}{2 \sinh r}
$$

proving part (ii).

For part (i) observe that the limit (1.2) can be expressed as an integral:

$$
\lim _{n \rightarrow \infty} \frac{1}{n} \sum_{i=1}^{n}\left(s_{i}-t_{i}\right)=\lim _{n \rightarrow \infty} \frac{1}{n} \int_{0}^{s_{n}} \chi_{\left(T_{1} B_{r}(q)\right)}\left(G_{v}(\tau)\right) d \tau .
$$

By Theorem 1 and the ergodic theorem for flows, this can be further divided into a product of limits, where each factor converges for almost all $v \in T_{1} S$. Thus (2.4) is equal to

$$
\begin{aligned}
& \lim _{n \rightarrow \infty} \frac{1}{s_{n}} \int_{0}^{s_{n}} \chi_{\left(T_{1} B_{r}(q)\right)}\left(G_{v}(\tau)\right) d \tau \times \lim _{n \rightarrow \infty} \frac{s_{n}}{n} \\
& =\frac{\operatorname{area}\left(B_{r}(q)\right)}{\operatorname{area}(S)} \times \frac{\operatorname{area}(S)}{2 \sinh r}=\frac{\pi(\cosh r-1)}{\sinh r}
\end{aligned}
$$

as asserted in part (i) of the Corollary, [1].

\section{Cone POINTS}

If the stabilizer of $i$ in $\Gamma$ is a subgroup of order $k>0$, then $q$ is called a cone point of order $k$. Let $R>0$ be the largest value so that for all $0<r<R$ the projection $\pi: B_{r}(i) \rightarrow B_{r}(q)$ is precisely $k-$ to -1 in the complement of $i$. Then for $0<r<R$, the definition of $N_{v}(r)(t)$ extends without modification to the case where $q$ is a cone point. The thickened sectioned is defined as before but now, since the map $\pi_{*}$ is $k-$ to -1 , instead of Proposition 1 we have

$$
\frac{1}{\epsilon} \mu\left(\mathcal{L}_{r}(\epsilon)\right)=\frac{1}{k \epsilon} \tilde{\mu}\left(\tilde{\mathcal{L}}_{r}(\epsilon)\right)=\frac{2 \sinh r}{k \operatorname{area}(S)} .
$$

Thus Theorem 1 remains valid when $q$ is a cone point of order $k$, with the right hand side of (1.1) replaced by (3.1).

When studying the rate of geodesic return to the neighborhood of a cone point, it is interesting to have all the quantities expressed in terms of area rather than radius. Let $D_{a}(q)$ denote the disc of area $a$ about $q$. Observe that, if $q$ is a cone point of order $k$, then the area of the $\operatorname{disc} B_{r}(q)$ is

$$
\operatorname{area}\left(B_{r}(q)\right)=\frac{1}{k} \operatorname{area}\left(B_{r}(i)\right)=\frac{2 \pi}{k}(\cosh r-1) .
$$

Thus $D_{a}(q)$ is a disc of radius $r=\cosh ^{-1}\left(\frac{k}{2 \pi} a+1\right)$.

Let $\left\{\left(t_{i}, s_{i}\right)\right\}$ be the sequence of excursions of the geodesic $\gamma=\gamma(v)$ into the disc $D_{a}(q)$. Then, almost as before, for $v \in \mathcal{E}$ we can define $\mathcal{N}_{v}(a)(t)=\max \left\{i \mid t_{i} \leq t\right\}$. 
In this setting, Theorem 1 for a cone point of order $k$, expressed in terms of area, takes the form:

Theorem 3. For almost all $v \in T_{1} S$ and all $0<a<A=(2 \pi / k)(\cosh R-1)$,

$$
\lim _{t \rightarrow \infty} \frac{1}{t} \mathcal{N}_{v}(a)(t)=\frac{2 \sqrt{\left(\frac{1}{k}+\frac{1}{2 \pi} a\right)^{2}+\frac{1}{k^{2}}}}{\text { area }(S)} .
$$

When $k$ is large, this value is close to $a /(\pi$ area $(S))$, which is the value computed in [5] for the asymptotic rate of return to a cusp neighborhood.

Also, the asymptotic average length of an excursion can still be computed when $q$ is a cone point. Replacing $B_{r}(q)$ by $D_{a}(q)$, equation (2.4) holds by definition and, as in equation (2.5), using Theorem 3 and the ergodic theorem we have the following.

Corollary 2. For almost all $v \in T_{1} S$ and all $0<a<A$, the average length of an excursion into $D_{a}(q)$ is

$$
\lim _{n \rightarrow \infty} \frac{1}{n} \sum_{i=1}^{n}\left(s_{i}-t_{i}\right)=\frac{a}{\operatorname{area}(S)} \times \frac{\operatorname{area}(S)}{2 \sqrt{\left(\frac{1}{k}+\frac{1}{2 \pi} a\right)^{2}+\frac{1}{k^{2}}}}=\frac{\pi}{\sqrt{\left(\frac{2 \pi}{k a}+1\right)^{2}+\left(\frac{2 \pi}{k a}\right)^{2}}} .
$$

For $k$ large, the average length of an excursion is close to $\pi$, which was the value computed in [5] for the average length of an excursion relative to a cusp neighborhood. When the area of the disc is large, the average length of an excursion is also close to $\pi$, independent of the order of the cone point.

\section{REFERENCES}

[1] A.F. Beardon, The Geometry of Discrete Groups, Springer-Verlag, Berlin, 1983. MR698777 (85d:22026)

[2] Ergodic Theory, Symbolic Dynamics and Hyperbolic Spaces, T. Bedford, H. Keane, C. Series, eds., Oxford Univ. Press, 1991. MR1130170 (93e:58002)

[3] I. P. Cornfeld, S.V. Fomin and Ya. G. Sinai, Ergodic Theory, Springer-Verlag, BerlinHeidelberg-New York, 1982. MR832433 (87f:28019)

[4] A. Haas, The distribution of geodesic excursions into the neighborhood of a cone singularity on a hyperbolic 2-orbifold, Comment. Math. Helv. 83, (2008), 1-20. MR2365405(2008k:37073)

[5] A. Haas, Geodesic cusp excursions and metric diophantine approximation, Preprint arxiv:0709.0313. To appear in Math. Res. Letters.

[6] H. Nakada, On metrical theory of Diophantine approximation over imaginary quadratic field. Acta Arith. 51 (1988), no. 4, 393-403. MR.971089 (89m:11070)

[7] P. Nicholls, The Ergodic Theory of Discrete Groups, Cambridge Univ. Press, 1989. MR1041575 (91i:58104)

[8] B. Stratmann, A note on counting cuspidal excursions. Ann. Acad. Sci. Fenn. Ser. A I Math. 20 (1995), no. 2, 359-372. MR1346819 (96k:58174)

Department of Mathematics, University of Connecticut, Storrs, Connecticut 06269

E-mail address: haas@math.uconn.edu 